\title{
RISK FACTORS OF MORTALITY IN NEONATAL ILLNESS
}

Jeyanthi Gandhi1, Poovazhagi Varadarajan ${ }^{2}$

${ }^{1}$ Assistant Professor, Department of Paediatrics, Tirunelveli Medical College Hospital.

2Professor of Paediatrics, Department of Paediatrics, Chengalpat Medical College Hospital.

\section{ABSTRACT}

\section{BACKGROUND}

Infant Mortality Rate (IMR) is high in India. Identification of risk factors of mortality in neonatal illness is essential to reduce Neonatal Mortality Rate (NMR) and ultimately the IMR.

\section{AIM}

To identify the risk factors of mortality in neonatal illness.

\section{SETTING AND DESIGN}

It was a nested case control study done at the sick neonatal unit of urban tertiary referral centre.

\section{METHODS AND MATERIALS}

After obtaining ethical committee approval, retrospective analysis of 150 out born neonatal case records of babies admitted during the period from October 2015 to December 2015 was done. Data such as demographic features, maternal details, referral details, perinatal events, clinical features, laboratory reports and outcome were recorded.

\section{STATISTICAL ANALYSIS}

These risk factors were subjected to univariate and multivariate logistic regression analysis and P value calculated for the same to find out significant risk factors of mortality in neonatal illness.

\section{RESULTS}

Neonatal mortality rate was $22 \%$. Male-to-female ratio was $2: 1$, death occurred more commonly in female neonates (23.1\%). Home deliveries carried more risk of mortality. Birth order 4 and above had 25\% mortality. Neonates of mother who had primary education and below had higher mortality. Perinatal asphyxia and sepsis were the most common causes of neonatal mortality. By univariate analysis, preterms had 4.9 times increased risk of mortality than term babies. Apnoeic spells, chest retractions and shock had 8 times, 3 times and 3.6 times increased risk of mortality respectively. By multivariate analysis, birth weight below 2 kilograms $(\mathrm{kg})$ carried 11.8 times more risk of mortality with a p value $0.00(95 \%$ C.I 3.2, 30.4) and poor maternal intake of iron and folic acid tablets was 3.9 times more risk p value 0.003 (95\% C.I 1.6, 9.6), apnoeic spells were 5.8 times more risk of mortality with p value $0.02(95 \%$ C.I $1.3,26.2)$.

\section{CONCLUSION}

Birth weight below $2 \mathrm{~kg}$, poor maternal intake of iron and folic acid tablets, apnoeic spells were significant risk factors of neonatal mortality.

\section{KEYWORDS}

Mortality, Newborn, Risk Factors.

HOW TO CITE THIS ARTICLE: Gandhi J, Varadarajan P. Risk factors of mortality in neonatal illness. J. Evolution Med. Dent. Sci 2016;5(20):1013-1016, DOI: 10.14260/jemds/2016/236

\section{INTRODUCTION}

Infant Mortality Rate (IMR) has shown a considerable fall from $80 / 1000$ live births (1991) to 40/1000 live births (2013) of the present, concerns have arisen due to stagnancy observed over last few years in the fall of IMR. This trend can be attributed to the relatively constant Neonatal Mortality Rate (NMR), which has hovered around 28/1000 live births. Unless efforts are concentrated on reducing NMR, it is difficult to reduce IMR. If neonatal mortality has to be reduced identification of causes of neonatal mortality is imperative, so as to put in place necessary intervention packages.

Financial or Other, Competing Interest: None.

Submission 25-01-2016, Peer Review 22-02-2016,

Acceptance 27-02-2016, Published 09-03-2016.

Corresponding Author:

Dr. Jeyanthi Gandhi,

3/L-49, TNHB Colony Phase 2,

Perumalpuram, Tirunelveli-627007,

Tamilnadu.

E-mail: drjeyanthi81@gmail.com

DOI: 10.14260/jemds/2016/236

\section{AIM}

To identify the risk factors of mortality in neonatal illness.

\section{MATERIALS AND METHODS}

It was a nested case control study done at sick neonatal unit of urban tertiary referral centre. Retrospective analysis of 150 cases records of all out born babies less than 28 days old, consecutively admitted during the period from October 2015 to December 2015 was done. Case records of newborn with surgical conditions, those who had discharges at request and against medical advice were excluded. Demographic factors such as gestational age, gender, place of referral, maternal educational status, parity, order of birth were listed. Information pertaining to delivery, immediate natal events were recorded. Symptomatology with which these babies presented, investigated, procedures, treatment modalities, final diagnosis and outcome were recorded.

Those who had death as outcome were considered as study cases, while those who recovered were taken as controls. 
Results were tabulated and simple percentage, proportions were derived for parameters such as gender, gestational age, diagnosis and neonatal mortality. Risk factors of mortality were identified by using univariate and multivariate analysis by using logistic regression. Odd's ratio for risk factors were calculated. P value less than 0.05 was considered significant; $95 \%$ confidence interval was also calculated for the same.

\section{RESULTS}

\begin{tabular}{|c|c|c|}
\hline & Number & $\mathbf{\%}$ \\
\hline Total number of case records screened & 150 & 100 \\
\hline $\begin{array}{c}\text { Total number of cases survived } \\
\text { (Controls) }\end{array}$ & 117 & 78 \\
\hline Total number of cases expired (Cases) & 33 & 22 \\
\hline Total number of males survived & 77 & 78.6 \\
\hline Total number of males expired & 21 & 21.4 \\
\hline Total number of females survived & 40 & 76.9 \\
\hline Total number of females expired & 12 & 23.1 \\
\hline
\end{tabular}

There are more number of males admitted than females with male:female ratio approaching 2:1. More number of deaths occurred in female (n:12, 23.1\%) when compared to males. As shown in Table 1, though majority of deliveries took place in institution (n:134, 90\%) about $10 \%(n=16)$ did take place at home of which $(n=6) 60 \%$ were preterm. Mortality for home delivery was high $(31.25 \%)$. First order deaths accounted for $51.51 \%$ of total deaths. Birth order beyond 3 was associated with $25 \%$ mortality. On the whole, $62.7 \%$ of term babies were admitted within the first 5 days of life, while
$75 \%$ of preterm babies were admitted within first $24 \mathrm{hrs}$. of birth.

Mortality below 5 days of age ranged between $18.7-20 \%$, while it was $40 \%$ if they were admitted beyond 5 days. Analysis of educational status of mothers revealed that $84 \%$ of them had only primary education and these mothers had high number of preterm deliveries and mortality was also high in their babies.

It was implied from Table 2, the most common cause of death was perinatal asphyxia $(n=8,24.2 \%)$ and sepsis $(n=8$, $24.2 \%)$ followed by respiratory distress ( $n=4,12.1 \%)$, congenital heart disease $(n=4,12.1 \%)$ respiratory distress syndrome $(n=4,12.1 \%)$ neonatal hyperbilirubinemia $(n=2$, $6 \%)$ preterm complications, congenital anomalies.

All 124 factors including maternal factors were analysed by univariate analysis and multivariate logistic regression to identify significant risk factors for mortality. On univariate analysis, risk factors such as birth weight less than 2 kilograms (kg) and 2 to $2.5 \mathrm{~kg}$, preterm, apnoeic spells, chest retractions, apnoea/gasping, shock and gastrointestinal disorders (Abdominal distention, diarrhoea, vomiting), poor intake of Iron and Folic Acid (IFA) tablets and multiple gestations were found to be significant as mentioned in Table 3 .

These univariate risk factors were then subjected to multivariate logistic regression analysis to identify risk factors, which were independently associated with mortality as indicated in Table 4 . Birth weight less than $2 \mathrm{~kg}$, poor maternal intake of iron and folic acid in antenatal period and apnoeic spells were found to be significant risk factors for mortality independent of each other.

\begin{tabular}{|c|c|c|c|c|c|c|c|}
\hline \multirow{2}{*}{ Sl. No. } & \multirow{2}{*}{ Factors } & \multicolumn{2}{|c|}{ Term } & \multicolumn{2}{|c|}{ Preterm } & \multicolumn{2}{|c|}{ Expired } \\
\hline & & Number & $\%$ & Number & $\%$ & Number & $\%$ \\
\hline 1. & $\begin{array}{c}\text { Gender } \\
\text { Male } \\
\text { Female }\end{array}$ & $\begin{array}{l}78 \\
40 \\
\end{array}$ & $\begin{array}{l}79.5 \\
76.9\end{array}$ & $\begin{array}{l}20 \\
12 \\
\end{array}$ & $\begin{array}{l}20.4 \\
23.1\end{array}$ & $\begin{array}{l}21 \\
12 \\
\end{array}$ & $\begin{array}{l}21.4 \\
23.1\end{array}$ \\
\hline 2. & $\begin{array}{l}\text { Place of Delivery } \\
\text { Institutional } \\
\text { Home }\end{array}$ & $\begin{array}{c}107 \\
10\end{array}$ & $\begin{array}{l}79.8 \\
62.5\end{array}$ & $\begin{array}{c}27 \\
6\end{array}$ & $\begin{array}{l}20.1 \\
37.5\end{array}$ & $\begin{array}{c}28 \\
5\end{array}$ & $\begin{array}{l}20.9 \\
31.3\end{array}$ \\
\hline 3. & $\begin{array}{c}\text { Order of Birth } \\
\\
1 \\
2 \\
3 \\
4\end{array}$ & $\begin{array}{c}55 \\
49 \\
10 \\
4\end{array}$ & $\begin{array}{l}77.5 \\
79.0 \\
76.9\end{array}$ & $\begin{array}{c}16 \\
13 \\
3\end{array}$ & $\begin{array}{c}22.5 \\
20.9 \\
23\end{array}$ & $\begin{array}{c}17 \\
13 \\
2 \\
1\end{array}$ & $\begin{array}{c}23.9 \\
20.9 \\
15.3 \\
25\end{array}$ \\
\hline 4. & $\begin{array}{c}\text { Day of Admission } \\
\begin{array}{c}<24 \text { hours } \\
1-5 \text { days } \\
>5 \text { days }\end{array}\end{array}$ & $\begin{array}{l}26 \\
74 \\
18\end{array}$ & $\begin{array}{c}52 \\
92.5 \\
90\end{array}$ & $\begin{array}{c}24 \\
6 \\
2\end{array}$ & $\begin{array}{l}48 \\
7.5 \\
10\end{array}$ & $\begin{array}{c}10 \\
15 \\
8\end{array}$ & $\begin{array}{c}20 \\
18.8 \\
40\end{array}$ \\
\hline 5. & $\begin{array}{c}\text { Maternal Educational } \\
\text { Status } \\
\text { Primary } \\
\text { High School } \\
\text { Graduation }\end{array}$ & $\begin{array}{c}98 \\
14 \\
6\end{array}$ & $\begin{array}{l}77.8 \\
82.4 \\
85.7\end{array}$ & $\begin{array}{c}28 \\
3 \\
1\end{array}$ & $\begin{array}{l}22.2 \\
17.6 \\
14.2\end{array}$ & $\begin{array}{c}29 \\
3 \\
1\end{array}$ & $\begin{array}{c}23 \\
17.6 \\
14.2\end{array}$ \\
\hline & & & 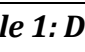 & aph & & & \\
\hline
\end{tabular}




\begin{tabular}{|c|c|c|c|c|c|c|c|c|c|c|c|}
\hline \multirow{3}{*}{$\begin{array}{l}\text { Sl. } \\
\text { No. }\end{array}$} & \multirow{3}{*}{$\begin{array}{c}\text { Causes } \\
\text { of Death }\end{array}$} & \multicolumn{4}{|c|}{ Term } & \multicolumn{4}{|c|}{ Preterm } & \multicolumn{2}{|c|}{ Total } \\
\hline & & \multicolumn{2}{|c|}{ Male } & \multicolumn{2}{|c|}{ Female } & \multicolumn{2}{|c|}{ Male } & \multicolumn{2}{|c|}{ Female } & \multirow[b]{2}{*}{ Admitted } & \multirow[b]{2}{*}{ Expired } \\
\hline & & Admitted & Expired & Admitted & Expired & $\begin{array}{c}\text { Admitte } \\
\text { d }\end{array}$ & Expired & Admitted & Expired & & \\
\hline 1. & $\begin{array}{l}\text { Perinatal } \\
\text { Asphyxia }\end{array}$ & 22 & 6 & 3 & - & 4 & - & 3 & 2 & $\begin{array}{c}\text { n } 32 \\
\% 21.33\end{array}$ & $\begin{array}{c}8 \\
24.2\end{array}$ \\
\hline 2. & Sepsis & 17 & 3 & 9 & 2 & 4 & 2 & 1 & 1 & $\begin{array}{c}\text { n } 31 \\
\% 20.6\end{array}$ & $\begin{array}{c}8 \\
24.2\end{array}$ \\
\hline 3. & $\begin{array}{c}\text { Respiratory } \\
\text { Distress }\end{array}$ & 13 & 2 & 5 & 2 & 1 & - & 2 & 2 & $\begin{array}{l}\text { n } 21 \\
\% 14\end{array}$ & $\begin{array}{c}4 \\
12.12\end{array}$ \\
\hline 4. & $\begin{array}{c}\text { Congenital } \\
\text { Heart } \\
\text { Disease }\end{array}$ & 5 & 1 & 3 & 2 & - & - & 1 & 1 & $\begin{array}{l}\text { n9 } \\
\% 6\end{array}$ & $\begin{array}{c}4 \\
12.12 \\
\end{array}$ \\
\hline 5. & $\begin{array}{l}\text { Respiratory } \\
\text { Distress } \\
\text { Syndrome }\end{array}$ & - & - & 1 & - & 5 & 4 & 1 & - & $\begin{array}{c}\mathrm{n} 7 \\
\% 4.6\end{array}$ & $\begin{array}{c}4 \\
12.12\end{array}$ \\
\hline 6. & $\begin{array}{c}\text { Neonatal } \\
\text { Hyperbiliru } \\
\text { binemia }\end{array}$ & 10 & 1 & 13 & - & 1 & 1 & 4 & - & $\begin{array}{c}\mathrm{n} 28 \\
\% 18.6\end{array}$ & $\begin{array}{c}2 \\
6.06 \\
\end{array}$ \\
\hline 7. & $\begin{array}{c}\text { Preterm } \\
\text { Complicatio } \\
n\end{array}$ & - & - & - & - & 3 & 2 & 1 & - & $\begin{array}{c}\mathrm{n} 4 \\
\% 2.6\end{array}$ & $\begin{array}{l}2 \\
6\end{array}$ \\
\hline 8. & $\begin{array}{l}\text { Congenital } \\
\text { Anomalies }\end{array}$ & 2 & - & 1 & - & 1 & 1 & - & - & $\begin{array}{c}\mathrm{n} 4 \\
\% 2.6 \\
\end{array}$ & $\begin{array}{c}1 \\
3.03 \\
\end{array}$ \\
\hline 9. & $\begin{array}{l}\text { Meconium } \\
\text { Aspiration } \\
\text { Syndrome }\end{array}$ & 2 & - & 1 & - & - & - & - & - & 3 & - \\
\hline 10. & Cholestasis & 3 & - & 1 & - & - & - & - & - & 4 & - \\
\hline 11. & $\begin{array}{c}\text { Physiologic } \\
\text { al } \\
\text { Conditions } \\
\text { (Transitiona } \\
\text { l stools) } \\
\end{array}$ & 2 & & 1 & - & 1 & - & - & - & 4 & - \\
\hline 12. & $\begin{array}{l}\text { Low Birth } \\
\text { Weight }\end{array}$ & 2 & - & - & - & - & - & - & - & 2 & - \\
\hline 13. & Seizures & 1 & - & - & - & - & - & - & - & 1 & - \\
\hline 14 & $\begin{array}{l}\text { Infant of } \\
\text { Diabetic } \\
\text { Mother }\end{array}$ & - & - & - & - & 1 & - & - & - & 1 & - \\
\hline & & & & & ible 2: & $\overline{\text { fDeath }}$ & & & & & \\
\hline
\end{tabular}

*N-Number

\begin{tabular}{|c|c|c|c|c|c|c|c|}
\hline $\begin{array}{l}\text { Sl. } \\
\text { No. }\end{array}$ & Factors & Admitted & \multicolumn{2}{|c|}{ Expired } & $\begin{array}{l}\text { Odd's } \\
\text { Ratio }\end{array}$ & $\begin{array}{l}\text { 95\% Confidence } \\
\text { Interval }\end{array}$ & P Value \\
\hline & Birth Weight & & Number & $\%$ & & & \\
\hline 1. & $\begin{array}{c}\quad<2 \mathrm{~kg} \\
2-2.5 \text { grams } \\
>2.5 \mathrm{~kg}\end{array}$ & $\begin{array}{l}35 \\
28 \\
69\end{array}$ & $\begin{array}{c}20 \\
3 \\
7\end{array}$ & $\begin{array}{l}57.1 \\
10.7 \\
10.1\end{array}$ & $\begin{array}{c}11.8 \\
1.1 \\
1\end{array}$ & $\begin{array}{c}4.2,33.1 \\
0.3,4.4\end{array}$ & $\begin{array}{l}0.00 \\
0.00\end{array}$ \\
\hline 2. & $\begin{array}{c}\text { Gestational Age } \\
\\
\text { Term } \\
\text { Preterm }\end{array}$ & $\begin{array}{c}118 \\
32\end{array}$ & $\begin{array}{l}18 \\
15\end{array}$ & $\begin{array}{l}15.3 \\
46.9\end{array}$ & $\begin{array}{c}1 \\
4.9\end{array}$ & $2.1,11.5$ & 0.00 \\
\hline 3. & $\begin{array}{c}\text { Clinical Profile } \\
\text { Apnoeic Spells } \\
\text { Cyanosis } \\
\text { Chest Retractions } \\
\text { Apnoea/Gasping } \\
\text { CRT } \\
\text { Shock } \\
\text { GI (abdominal distention, } \\
\text { diarrhoea) }\end{array}$ & $\begin{array}{l}16 \\
11 \\
34 \\
11 \\
19 \\
32 \\
10\end{array}$ & $\begin{array}{c}10 \\
6 \\
13 \\
7 \\
8 \\
5 \\
1\end{array}$ & $\begin{array}{c}62.5 \\
54.5 \\
38.2 \\
63.6 \\
42.1 \\
19.1 \\
10\end{array}$ & $\begin{array}{c}8 \\
- \\
3 \\
7.6 \\
- \\
3.6 \\
2.8\end{array}$ & $\begin{array}{c}2.7,24.3 \\
- \\
1.3,6.9 \\
2.1,27.9 \\
3.1 \\
1.4,9.7 \\
1.2,6.5\end{array}$ & $\begin{array}{c}0.00 \\
0.02 \\
0.01 \\
0.002 \\
0.03 \\
0.001 \\
0.02\end{array}$ \\
\hline 4. & $\begin{array}{c}\text { Poor intake of iron and folic acid } \\
\text { tablets }\end{array}$ & 27 & 12 & 44.4 & 3.9 & $1.6,9.6$ & 0.003 \\
\hline 5. & Multiple Gestation & 9 & 5 & 55.6 & 4.7 & $12,18.8$ & 0.03 \\
\hline
\end{tabular}




\begin{tabular}{|c|c|c|c|c|c|c|c|}
\hline $\begin{array}{l}\text { Sl. } \\
\text { No. }\end{array}$ & Factors & Admitted & \multicolumn{2}{|c|}{ Expired } & $\begin{array}{l}\text { Odd's } \\
\text { Ratio } \\
\end{array}$ & $\begin{array}{c}95 \% \\
\text { C.I } \\
\end{array}$ & P Value \\
\hline \multirow[b]{2}{*}{1.} & Birth Weight & & Number & $\%$ & & \multirow[b]{2}{*}{$\begin{array}{c}4.2,33.1 \\
0.3,4.4\end{array}$} & \multirow[b]{2}{*}{0.00} \\
\hline & $\begin{array}{c}<2 \mathrm{~kg} \\
2-2.5 \mathrm{~kg} \\
>2.5 \mathrm{~kg}\end{array}$ & $\begin{array}{l}35 \\
28 \\
69 \\
\end{array}$ & $\begin{array}{c}20 \\
3 \\
7 \\
\end{array}$ & $\begin{array}{l}57.1 \\
10.7 \\
10.1 \\
\end{array}$ & $\begin{array}{c}11.8 \\
1.1 \\
1 \\
\end{array}$ & & \\
\hline 2. & $\begin{array}{l}\text { Poor intake of iron and folic acid } \\
\text { tablets }\end{array}$ & 27 & 12 & 44.4 & 3.9 & $1.6,9.6$ & 0.003 \\
\hline 3. & Apnoeic Spells & 16 & 10 & 62.5 & 5.8 & $1.3,26.2$ & 0.02 \\
\hline
\end{tabular}

*C.I Confidence Interval

\section{DISCUSSION}

India is fast achieving a state of a developed country. Good health indices are a must for that state. The current trends of neonatal and of maternal mortality are not in favour of India achieving that status in the near future. It is of great concern that IMR has remained practically constant in the last few years, so that India could not achieve Millennium development goal of $27 / 1000$ live births by 2015 . This is due to the slow rate of decline of neonatal mortality, which contributes to $70 \%$ of the infant mortality.(1) Efforts to reduce neonatal mortality require that the cases of neonatal mortality be assessed in its proper perceptive. This study was designed to understand factors affecting neonatal mortality and to identify significant risk factors for neonatal mortality.

Neonates usually present with constellation of symptoms rather than unique symptoms pointing to particular disease. They also manifest with more than one disease. Against this background, this study was focused on identification of significant risk factors of neonatal mortality, which are common irrespective of disease profile. Studies all over the world pointed out that low birth weight neonates have higher mortality.(2) Birth asphyxia and sepsis constitute most common causes of neonatal mortality.(3) First order birth, birth order beyond 3 carried increased risk of mortality. Although Government devised programme to institutionalize deliveries which seems to be working well in the state, still home deliveries do occur and mortality remains high in such situations.(4) Maternal education does have an important role in neonatal mortality.
This study has shown that decreasing educational background is associated with increasing neonatal mortality. This can be evidenced by low IMR at Kerala, where female literacy rate is maximum.(5) Poor maternal intake of iron and folic acid tablets and thereby anaemia increases the risk of neonatal mortality.

\section{CONCLUSION}

Birth weight less than $2 \mathrm{~kg}$, poor maternal intake of iron and folic acid tablets, apnoeic spells were significant risk factors of neonatal mortality.

\section{REFERENCES}

1. Joshi HP, Bhavisha, Kadambari Singh. An analysis of levels and trends in infant and child mortality rates in India. New Delhi: National institute of public cooperation and child development. 2014;11-24.

2. Bang AT, Bang RA, Baitule S. Burden of mortalities and the unmet need for health care in rural neonates-a prospective observational study in Gadchiroli, India. Indian Pediatr 2001;38:952-965.

3. Zodpey S, Paul VK, Neogi BS, et al. State of India's newborns 2014. New Delhi: Public health foundation of India and all India institute of medical sciences. 2014;4-19.

4. Narang M, Sharma KJ, Sharma AK, et al. Predictors of mortality among the neonates transported to referral centre in Delhi. Indian J Public Health 2013;57:100-4.

5. Upadhyay RP. High neonatal mortality rates in rural India: what options to explore? ISRN Pediatrics 2012;2012:9-10. 